\title{
Application du filtrage spatial à l'analyse des contours des zones anomales de II dérangements II des séries phosphatées de Sidi Chennane, Maroc
}

\section{Saad BAKKALI}

Département des sciences de la terre, Faculté des sciences et techniques, BP 416 TANGER, MAROC

(Reçu le 25 Septembre 2005, accepté le 11 Janvier 2006)

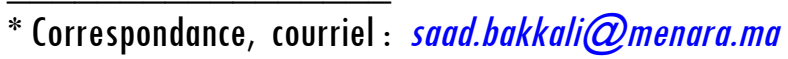

\section{Résumé}

Situé dans le grand bassin des Oulad Abdoun, le gisement de Sidi Chennane a été retenu pour prendre la relève du gisement du Grand Daoui. Dans ce gisement phosphatier, des discontinuités appelées communément $\|$ dérangements $\|$ affectent totalement et/ou partiellement la succession habituelle de la série phosphatée. Ces "dérangements ॥ perturbent considérablement les chaînes cinématiques d'exploitation. La résistivité des "I dérangements "I est comprise entre 200 et $1000 \Omega$.m. Couvrant un panneau de $50 \mathrm{Ha}$, cette étude pilote avait pour objectif de cartographier et de délimiter en surface les zones anomales correspondant aux "dérangements ॥. Une carte de résistivités apparentes avait été réalisée avec un dispositif Schlumberger afin de circonscrire les zones anomales. Dans ce présent travail, nous présentons les résultats de l'analyse des contours des zones de II dérangements " par utilisation directe des techniques usuelles du filtrage spatial. Les opérateurs II enhancement edge "I et laplacien-filtre passe-haut ont été utilisés. Ces procédés ont permis de caractériser spatialement les anomalies géoélectriques tout en amoindrissant leurs caractères aléatoires. Les estimations de calcul des réserves se trouvent fortement circonscrites.

Mots- clés : Résistivité, phosphates, filtrage, contours, Sidi Chennane, Maroc.

\section{Abstract}

Space filtering applied to the analysis of anomalous zones edges of phosphate "disturbances" of Sidi Chennane, Morocco

In the Oulad Abdoun phosphate basin, discontinuities called commonly "disturbances" affect completely and/or partially the usual succession of the phosphatic series. 
Generally very hard and detectable only at the boring, these "disturbances" disturb considerably the kinematic chains of exploitation. Calculations of the reserves are distorted. The resistivity of the "disturbances" lies between 200 and $1000 \Omega$.m. In this specific context, a geophysical survey by geoelectric prospection had been carried out. Covering a panel of $50 \mathrm{Ha}$, this study controls had allowed to map and delimit on the surface the anomalic zones corresponding to the "disturbances". Measurements of apparent resistivity had been acquired with a Syscal2 resistivity meter of BrgmInstruments. We propose in this present study the contribution of spatial analysis for a better interpretation of the Sidi Chennane phosphate "disturbances" map. Operators in the spatial domain like enhancement edge and laplacian high-pass filter are applied to the phosphate anomalous zones.

Keywords : Resistivity, phosphate, edge, filtering, Sidi Chennane, Morocco.

\section{Introduction}

Avec une capacité de production de 19 millions de tonnes par an, et plus de 35 milliards de $\mathrm{m}^{3}$ de réserves, le Maroc détient actuellement plus de $75 \%$ des réserves mondiales des phosphates. Dans ce contexte spécifiquement industriel, la prospection géophysique par la méthode des résistivités électriques a été appliquée dans le bassin phosphatier Oulad Abdoun dans la province de Khouribga (Figure 1 ) située à près de $120 \mathrm{~km}$ au Sud - Est de la capitale économique Casablanca.

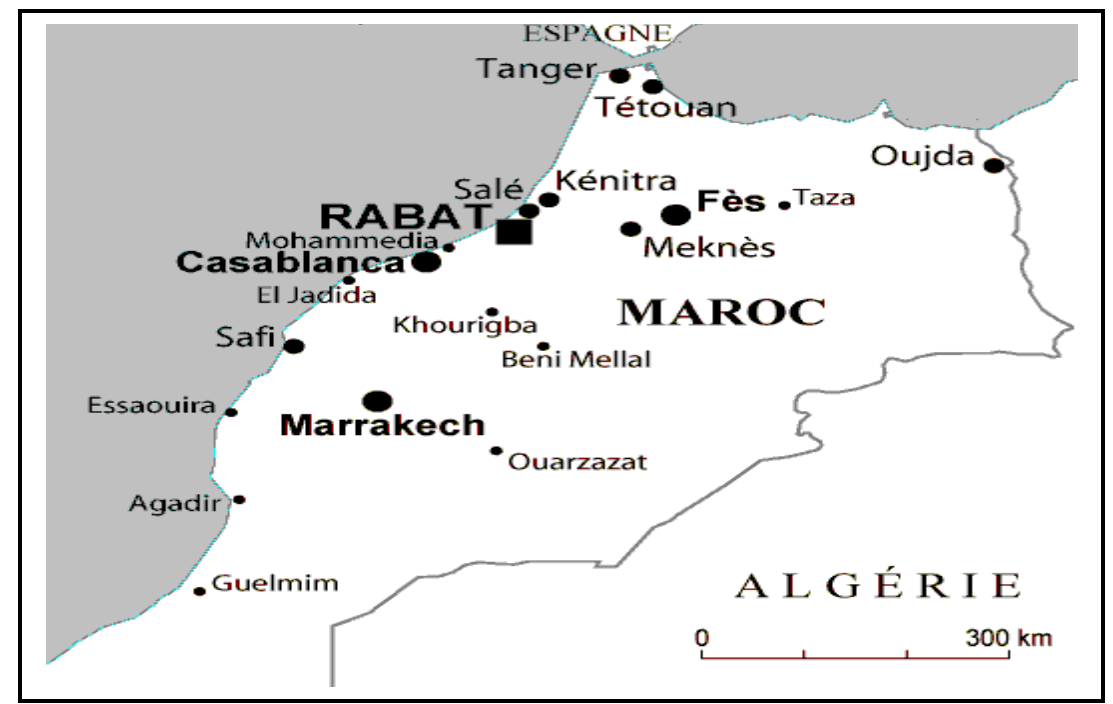

Figure 1 : Carte de la situation géographique de la zone d'étude 
L'étude géophysique avait pour cadre spécifique le gisement de Sidi Chennane, l'une des parcelles du bassin sédimentaire Oulad Abdoun. Le gisement de phosphate est de type sédimentaire, présentant plusieurs couches phosphatées. Les couches phosphatées alternent avec des niveaux de marnes et de calcaires. Le gisement de Sidi Chennane a été retenu pour prendre la relève du gisement dit du Grand Daoui. Au sein du gisement des discontinuités appelées $\|$ dérangements "I affectent totalement et/ou partiellement la succession habituelle de la série phosphatée. Stériles, généralement très durs et détectables uniquement lors de la foration, les II dérangements " perturbent les chaînes cinématiques d'exploitation en biaisant les calculs de réserve. Les méthodes directes telles les coupes de puits et les indices de surface s'étaient avérées insuffisantes et non adaptées pour la reconnaissance de ces discontinuités [1].

La résistivité de la série phosphatée normale est comprise entre 80 et $150 \Omega$.m; celle des I dérangements " est comprise entre 200 et plus de $1000 \Omega$.m. Dans ce contexte particulier une étude géophysique pilote de prospection électrique en courant continu avait été réalisée. Couvrant un panneau de $50 \mathrm{Ha}$, cette étude pilote avait pour objectif de cartographier et de délimiter en surface les zones anomales correspondant aux " dérangements ॥ [2]. La carte de résistivité fournit une interprétation directe et imagée des séries de profils de traînées électriques dont elle découle. Dans notre cas une carte de résistivité apparente fut réalisée. Le dispositif utilisé fut un dispositif Schlumberger [2] centré de longueur de ligne $A B=120 \mathrm{~m}$. Ce procédé avait permis d'identifier les zones anomales coïncidant avec les I dérangements " pour des profondeurs moyennes inférieures à $40 \mathrm{~m}$ [2]. Les II dérangements II se présentent de façon aléatoire.

La carte joue le rôle fondamental de II radar " pour les conducteurs de travaux, et ce afin de ne pas briser la châne cinématique d'exploitation. Les techniques classiques du filtrage spatial servent alors à circonscrire efficacement les effets aléatoires de la distribution des "dérangements " correspondant aux anomalies géoélectriques. Une étude comparative des différentes réponses du filtrage spatial appliqué à la carte des " dérangements " permettra aux conducteurs de travaux d'exploiter adéquatement les séries phosphatées saines et non ॥ dérangées ॥.

\section{Présentation de la zone d'étude}

\section{2-1. Géographie de la zone}

Le gisement de Sidi Chennane du bassin phosphatier Oulad Abdoun est localisé à $33 \mathrm{~km}$ au Sud - Est de Khouribga (Figure 2). Il est délimité à l'ouest par le méridien Lambert 372500 au sud par le méridien 228 000, à l'est par la route principale RP22 et au nord par les affleurements de la base de la série phosphatée. Le plateau des phosphates est 
essentiellement une région à climat aride. La pluviométrie, étalée sur les mois allant de novembre à mai, ne dépasse généralement guère les $400 \mathrm{~mm}$, ne permettant ainsi qu'une végétation clairsemée principalement représentée par des palmiers nains. La population rurale, vivant particulièrement d'élevage et de cultures saisonnières est dispersée dans de petits villages ou douars. Les besoins en eau de cette région sont de plus en plus pressants, et les manques y sont surtout enregistrés avec une certaine gravité. Exceptés quelques puits aléatoirement éparpillés alimentés par les infiltrations au cours de la période hivernale, et dont les marnes sénoniennes forment l'écran imperméable, le seul réservoir aquifère est représenté par le calcaire turonien. Ce dernier situé généralement à plus de $100 \mathrm{~m}$ de profondeur, constitue la roche réservoir la plus importante de la région, jouant ainsi un rôle déterminant dans l'alimentation en eau de divers centres d'exploitation.

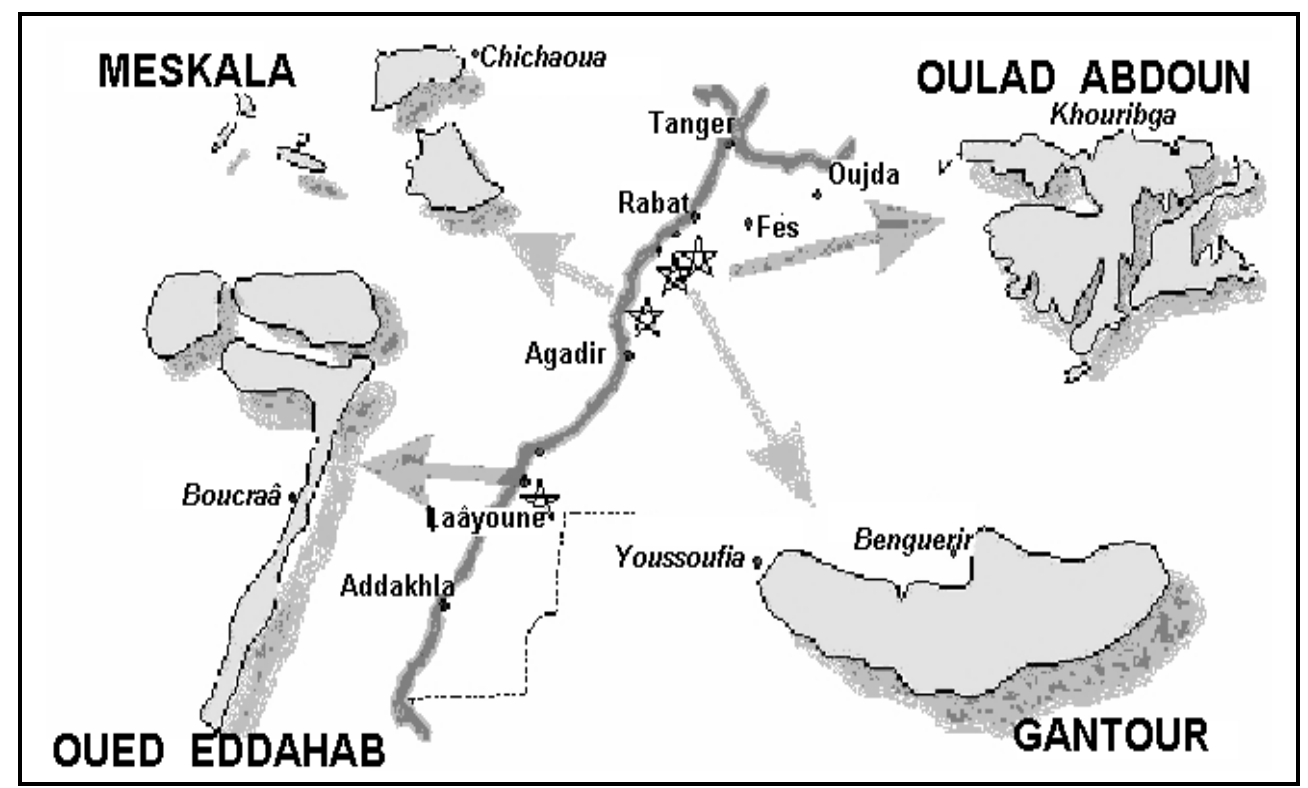

Figure 2 : Carte des bassins des phosphates du Maroc

\section{2-2. Aperçu géologique}

Les phosphates ont été déposés sur une longue période allant de la fin du Crétacé (étage du Maestrichtien, environ 80 Millions d'années) jusqu'au début de l'Eocène (étage du Lutétien basal ou Lutétien inférieur 40 Millions d'années). Néanmoins entre ces périodes les dépôts n'étaient pas constants. II manque par conséquent des sédiments : ce qui atteste de la discontinuité de la chaîne d'information géologique. Le bassin des Oulad 
Abdoun couvre la partie principale de l'élément morphologique appelé plateau des phosphates. II est borné au Nord par les affleurements des dépôts rouges infracénomaniens qui prolongent le flanc sud du massif central, à l'Ouest par le massif des Rhamna, au Sud par la plaine de Beni Amir et à l'est par le haut Atlas de Béni Mellal. Géologiquement, la série de ce plateau des phosphates auquel est identifiée la région de notre site d'étude est bien étudiée et caractérisée par la coupe stratigraphique de la Figure 3.

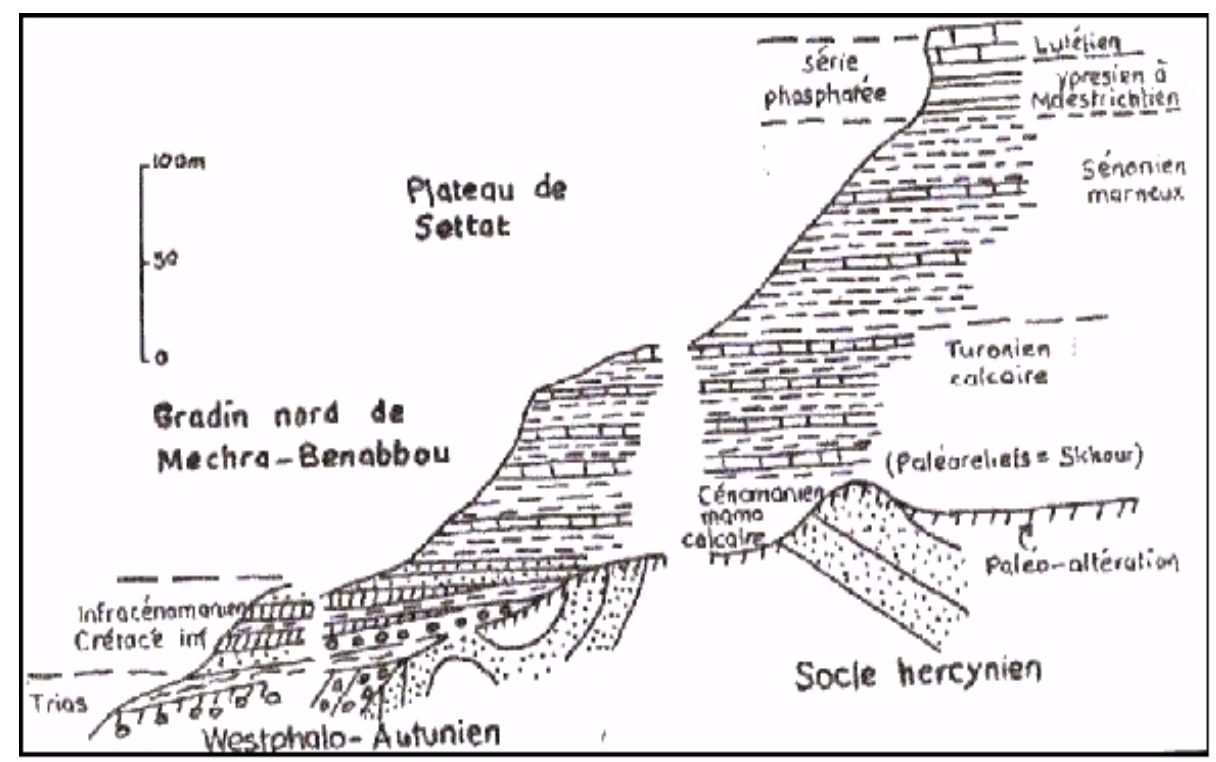

Figure 3 : Coupe stratigraphique du socle et de la couverture

Toutes les formations reposent par discordance sur les schistes et les quartzites du Primaire. Nous avons un socle bien marqué et une couverture plus ou moins épaisse [3]. Au sommet le Maastrichtien et l'Eocène qui constituent la série phosphatée d'une puissance de 30 à $50 \mathrm{~m}$, le Maastrichtien $(5$ à $28 \mathrm{~m}$ ) est représenté par des phosphates marneux tandis que l'Eocène (20 à $30 \mathrm{~m}$ ) est représenté par des faciès plus variés tels les marnes phosphatées et les phosphates sableux avec intercalations calcaires. En dessous suivent respectivement :

- le Sénonien, épais de 40 à 70 m, est formé d'un ensemble de marnes et de marnes calcaires.

- le Turonien calcaire d'épaisseur de 20 à $60 \mathrm{~m}$.

- le Cénomanien représenté par l'alternance de marnes souvent gypseuses et de marnes calcaires. 
- l'Infracénomanien ( 10 à $60 \mathrm{~m}$ ) et formé de marnes bariolées et de grès rouges.

Les I dérangements " objet de cette étude associent les paramètres tels l'ampleur, la nature des matériaux, la dureté, la teneur en argile, la nature du contact "dérangement " - série normale. Les "dérangements " ont été singulièrement différenciés selon 2 types prédominants :

- Le premier type affecte toute la série phosphatée correspond aux II dérangements ॥ formés d'un mélange hétéroclite de calcaires, de marnes, d'argiles, de silex et phosphate avec abondance de calcaire riche en silex.

- Le second type représente une série totalement perturbée sans prédominance de lithofaciès. Le II dérangement II se présente sous forme d'un amas de blocs de calcaires phosphatés à gros nodules de silex, des marnes, des argiles, des portions de bancs de silex et des phosphates. (e type de II dérangement II dont les dimensions ont des diamètres variant de 10 à plus de $150 \mathrm{~m}$, est le plus fréquemment rencontré lors de l'exploitation (Figure 4).

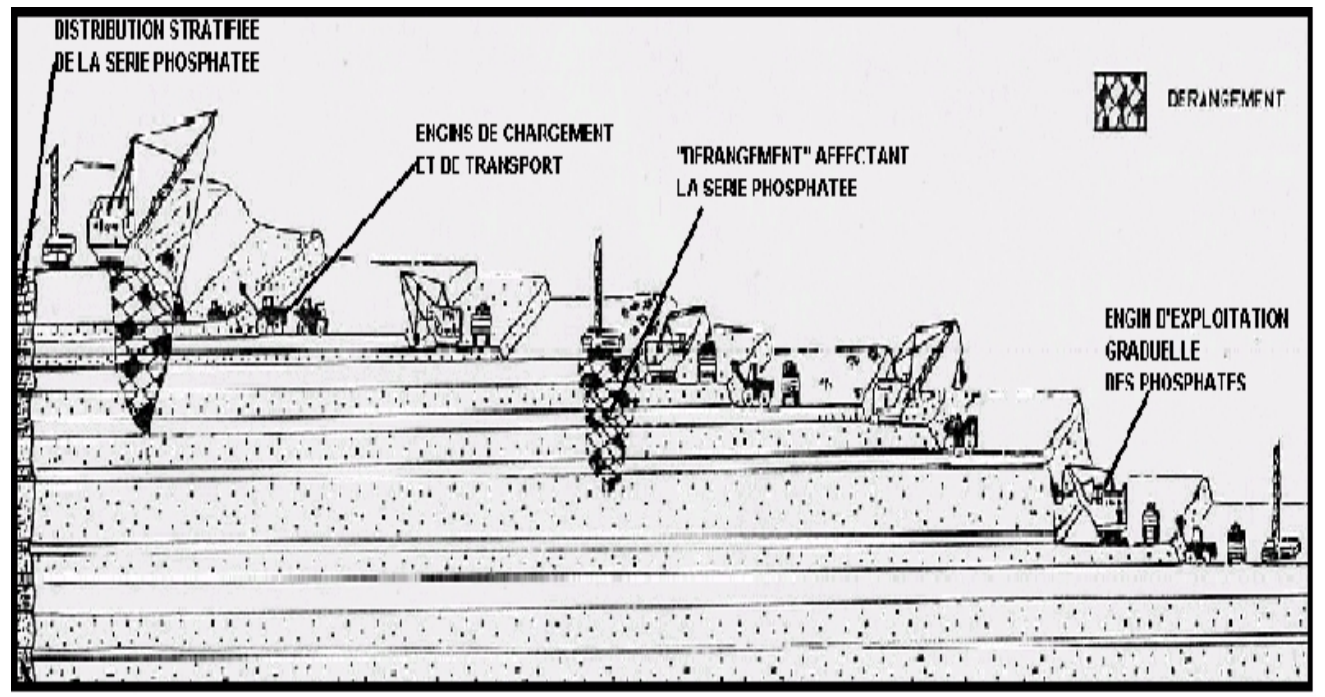

Figure 4 : Impact des II dérangements ॥ sur la chaîne d'exploitation

Il affecte le primaire stérile et les termes supérieurs de la série phosphatée. Ces particularités se traduisent par un fort contraste de résistivité entre les " dérangements" et la série phosphatée normale telle que confirmée par les profils -étalon de résistivité (Figure 5 ). La campagne de prospection géophysique a été ainsi basée sur la mise en évidence de ces contrastes de résistivité sur l'ensemble du panneau de cette étude pilote. 


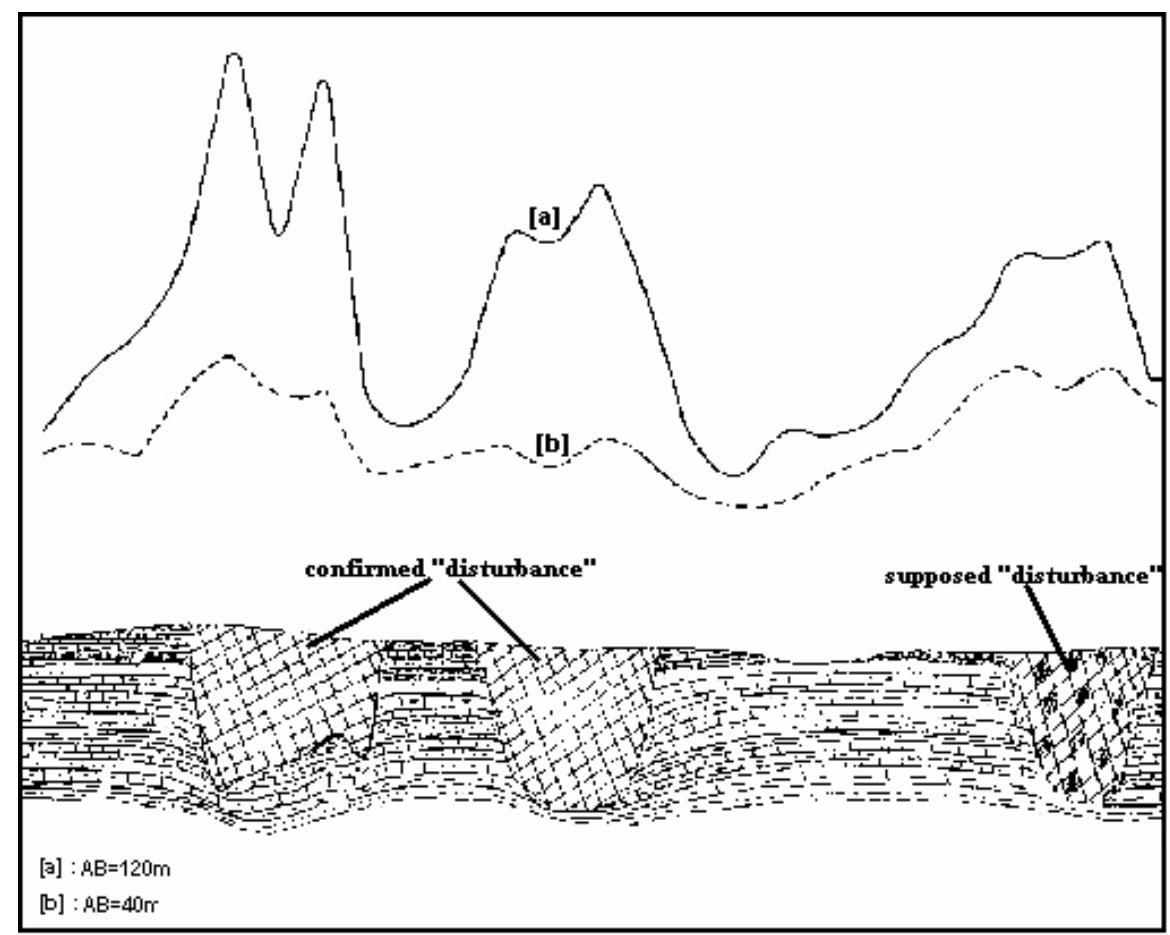

Figure 5 : Profils de résistivités sur II dérangements ॥

\section{Méthodologie}

\section{3-1. Matériel et méthodes}

La méthode du trâ̂née électrique a été utilisée pour délimiter les zones anomales correspondantes aux II dérangements $॥$. Le principe général de la prospection électrique par traînés électriques repose sur l'injection d'un courant électrique I entre deux électrodes $A$ et $B$ (dites de courant) et la mesure d'une différence de potentiel $\Delta \mathrm{V}$ entre deux autres électrodes $M$ et $N$ (dites de potentiel). $0 n$ en déduit la résistivité apparente du sous-sol:

$$
\rho_{\text {apparent }}=K \frac{\Delta V}{I}
$$

où $K$ est le coefficient géométrique du dispositif, fonction uniquement des distances entre électrodes [4]. Le rapport des deux paramètres I et $\Delta \mathrm{V}$ permet de calculer la résistivité des terrains sous-jacents. Le dispositif Schlumberger utilisé dans ce travail nécessite que les électrodes soient alignées, symétriques par rapport à leur centre 0 avec

\section{Saad Bakkali}


$M N<<A B[5,6]$. Les traînées électriques permettent d'obtenir des profils et des cartes de résistivités apparentes. Plus la longueur de ligne sera grande, plus la profondeur d'investigation augmentera. Les traînées électriques de résistivité apparente rendent compte de la variabilité horizontale des propriétés du sous-sol $[5,6]$. Ils servent à déterminer l'extension surfacique des couches en une position donnée, et permettent de confirmer la présence ou l'absence des II dérangements II. Ils permettent également de circonscrire les variations latérales de faciès.

Les mesures de résistivité apparente ont été réalisées avec le résistivimètre Syscal2 de Brgm-Instruments aux sommets d'une maille rectangulaire de dimensions $20 \mathrm{~m} \times 5 \mathrm{~m}$. Pour couvrir l'ensemble de la zone d'étude et afin de cibler des profondeurs de "I dérangements ॥ moyennes de $40 \mathrm{~m}, 51$ profils de traînées électriques régulièrement espacés de $20 \mathrm{~m}$ ont été exécutés. Chacun des profils électriques représente 101 stations de mesure équidistantes de $5 \mathrm{~m}$. L'équidistance entre les profils est de $20 \mathrm{~m}$. L'ensemble de cette étude pilote avait représenté ainsi plus de 5151 mesures de résistivité apparente (Figure 6) [2].

DIRECTION NORD (DISTANCE EN METRES)

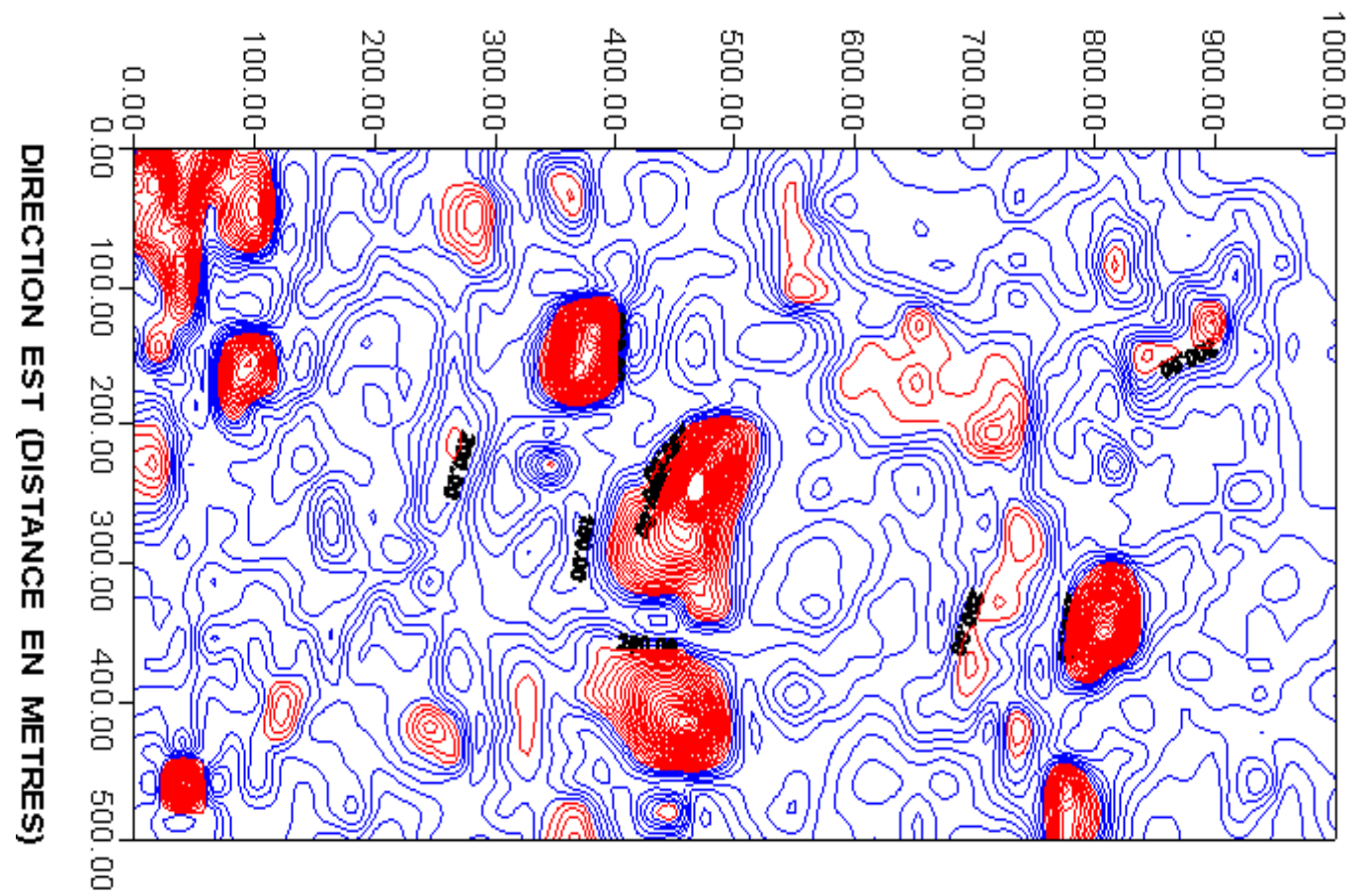

Figure 6 : Carte des résistivités apparentes de la zone d'étude (intervalle contour : $20 \Omega$.m). Les zones de "dérangements" correspondent aux résistivités anomales (iso-courbes rouges de résistivité $\geq 200 \Omega$.m) 


\section{3-2. Données géophysiques}

L'interprétation directe des caractéristiques d'une anomalie géoélectrique permet d'obtenir rapidement différentes informations qualitatives et semi-quantitatives sur la nature et la position du corps perturbateur. Le corps perturbateur est supposé constitué essentiellement de II dérangements I. Les II dérangements II sont les corps potentiants. L'amplitude est proportionnelle au volume du corps perturbateur et au contraste de résistivité entre celui-ci et les roches encaissantes. Une structure n'apparaîtra pas si les roches qui la constituent ont la même résistivité que le terrain encaissant. La résistivité apparente découle d'un potentiel harmonique $\mathrm{V}$ vérifiant l'équation de Laplace $\Delta \mathrm{V}=0$ en dehors des corps potentiants, et dont le gradient de celui-ci est du type $1 / \mathrm{r}^{2}$. En première approche, il est supposé qu'au ॥ voisinage ॥ ainsi que dans les sources représentées par les II dérangements II le potentiel scalaire vérifie l'équation

$$
\Delta V=-2 \rho I \delta(r)
$$

ou $\delta(r)$ est la distribution de Dirac, $\rho$ est la résistivité de la zone anomale et I est le courant injecté par une électrode ponctuelle sur un demi-espace élastique (surface topographique de la zone d'étude) [7]. La cartographie géoélectrique réalisée par mesures des résistivités apparentes sur une grille régulière est en fait une cartographie des potentiels discrets mesurés en surface. La singularité des fortes valeurs de résistivités apparentes sur les zones des II dérangements I résulte à juste titre du passage d'une zone saine à une zone "dérangée II. Sous ces hypothèses, la carte de résistivité apparente est en fait par analogie une carte de différences de potentiel scalaire supposées partout harmoniques excepté dans les zones II dérangées II [8].

La carte des résistivités anomales permet une définition physique des sources. La carte de différences de potentiel s'adonne ainsi usuellement au traitement numérique des données dans le domaine spatial [8]. Nous avons fait subir à la carte des zones anomales de "dérangements" correspondante à la carte des résistivités anomales, qui est une image numérique (Figure 7), plusieurs filtrages dans le domaine spatial. Ces signatures spatiales, qui ne sont autres que des filtrages numériques, permettent de circonscrire géométriquement les surfaces anomales correspondant fortement aux " dérangements ॥[9]. 


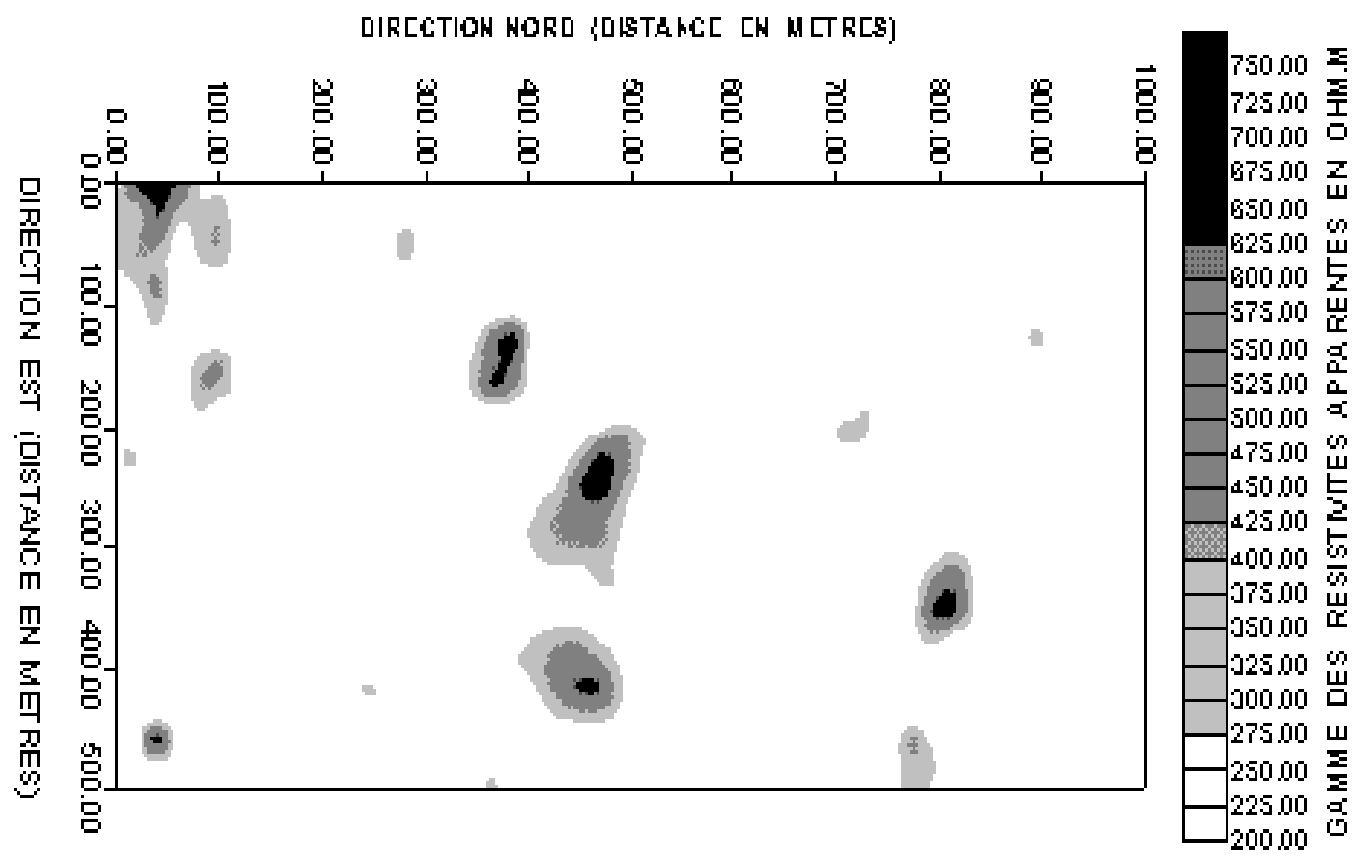

Figure 7 : Carte de distribution des zones anomales de "dérangements"

\section{3-3. Procédé de traitement}

La carte des zones de " dérangements $\|$ est représenté par une image numérique $I(x, y): R x R \rightarrow R$ considérée comme une matrice de points appelés pixels repérés par leurs coordonnées $(x, y)$. A chacun d'entre eux est associé une valeur correspondant à la mesure d'un phénomène en ce lieu, qui en l'occurrence, est représenté par la résistivité apparente.

Le filtrage spatial consiste à mélanger localement les informations mesurées (la valeur résultante d'un pixel est une fonction de tout le voisinage de ce pixel). Les filtres spatiaux représentent une méthode de traitement numérique utilisée pour le rehaussement d'une image. Ces filtres sont conçus de façon à faire ressortir ou à supprimer des caractéristiques spécifiques d'une image en se basant sur leur fréquence spatiale [10]. La fréquence spatiale est liée au concept de texture. Elle fait référence à la fréquence de variation des différents tons qui apparaissent dans une image, lesquels sont intimement liés aux données mesurées. Le filtrage spatial numérique consiste à appliquer une transformation (appelée filtre) à tout ou partie d'une image numérique en appliquant un opérateur. 


\section{3-4. Filtre laplacien}

Le laplacien-filtre passe-haut est une technique usuelle permettant de souligner les détails d'une carte d'anomalies. C'est un filtre de convolution particulier utilisé pour mettre en valeur les détails qui ont une variation rapide de luminosité. En admettant que les variations brutales des changements de densité relevés sur les "dérangements $"$ induisent des variations brutales de luminosié, le laplacien-filtre est donc idéal pour circonscrire les contours des objets assimilés aux II dérangements " (Figure 8 ). Cette méthode, basée sur la dérivée seconde, permet de souligner les anomalies de courte longueur d'onde (haute fréquence) [11]. D'un point de vue mathématique le laplacienfiltre est une dérivée d'ordre 2 à 2 dimensions où son expression :

$$
\frac{\partial^{2}}{\partial x^{2}}+\frac{\partial^{2}}{\partial y^{2}}
$$

L'image de départ $I(x, y)$ n'étant pas une fonction continue mais discrète à cause de la numérisation, la dérivée est obtenue par différences finies.

L'amplitude $|\boldsymbol{L}(\boldsymbol{x}, \boldsymbol{y})|$ du laplacien-filtre passe-haut, en une côte constante $\mathrm{z}=\mathrm{z}_{0}$ correspondant à la surface topographique d'étude, est définie dans le domaine spatial par :

$$
|\boldsymbol{L}(\boldsymbol{x}, \boldsymbol{y})|=\left|\frac{\partial^{2} \boldsymbol{I}(\boldsymbol{x}, \boldsymbol{y})}{\partial \mathrm{x}^{2}}+\frac{\partial^{2} \boldsymbol{I}(\boldsymbol{x}, \boldsymbol{y})}{\partial y^{2}}\right|
$$

Les dérivées horizontales sont habituellement calculées par la méthode des différences finies par les expressions suivantes:

$$
\frac{\partial \boldsymbol{I}(\mathrm{x}, \mathrm{y})}{\partial \mathrm{x}}=\frac{\boldsymbol{I}_{\mathrm{i}+1, j}-\boldsymbol{I}_{\mathrm{i}-1, j}}{2 \delta \mathrm{x}} \quad \text { et } \quad \frac{\partial \boldsymbol{I}(\mathrm{x}, \mathrm{y})}{\partial \mathrm{y}}=\frac{\boldsymbol{I}_{\mathrm{i}, j+1}-\boldsymbol{I}_{1, j-1}}{2 \delta \mathrm{y}}
$$

ou $I_{i, j}$ est le pixel correspondant à la pseudo-résistivté apparente $\rho_{i, j}$ définie au nœud (i,j) de la grille de mesures aux pas respectifs $\delta x$ et $\delta y$ dans les directions Nord et Est. La dérivée d'ordre deux issue du laplacien-filtre est une opération de dérivation qui correspond à un filtrage passe-haut. Cette opération de dérivation, usuellement réalisée dans le domaine de Fourier, représente également un moyen principal du procédé d'interprétation parce qu'elle permet d'affiner la réponse des dispositifs géophysiques: elle permet de mieux définir les contours. L'opérateur du laplacien-filtre passe-haut souligne le mieux l'effet des structures et discontinuités superficielles puisque le calcul des dérivées y est particulièrement sensible [12]. 
DIRECTION MORD (DISTANCE EN ME TRES

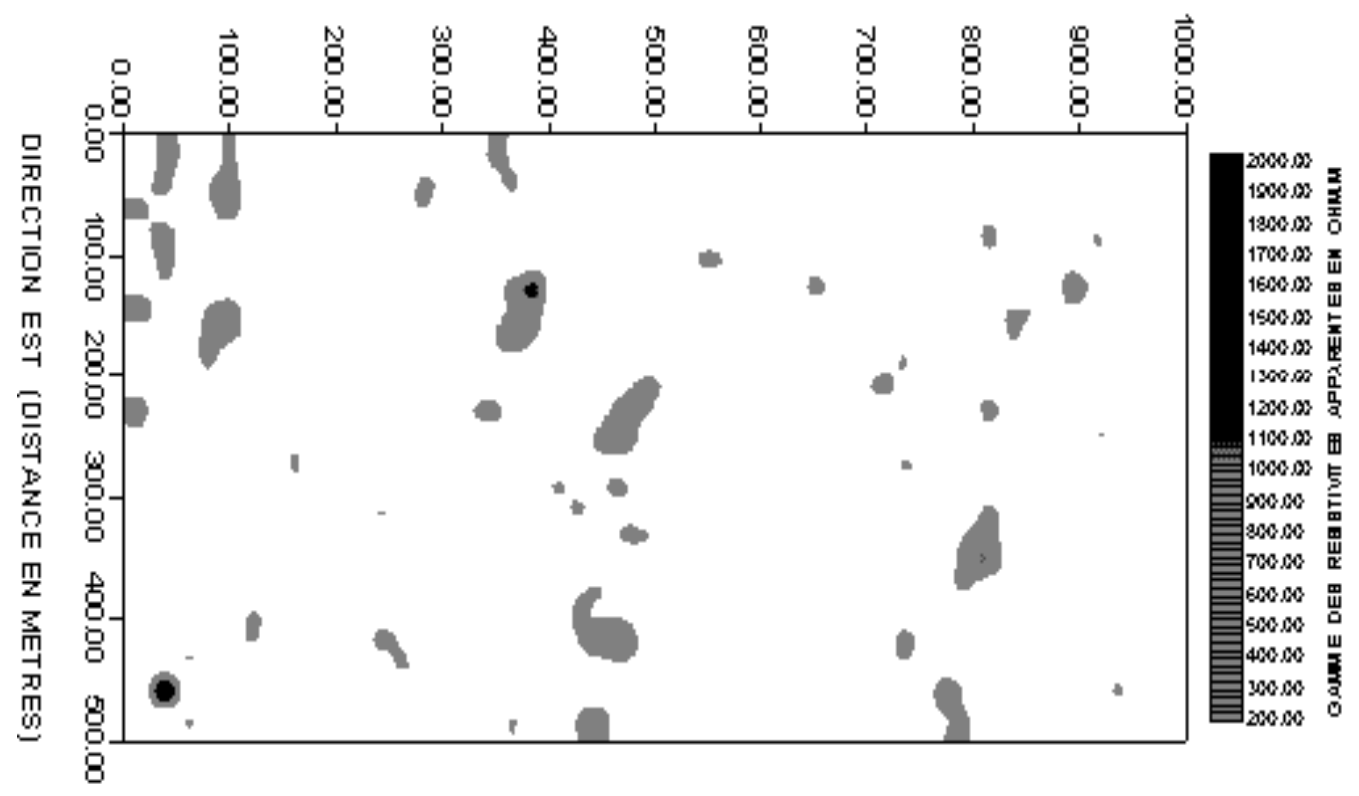

Figure 8 : Réponse du laplacien-filtre passe-haut appliqué aux zones anomales de II dérangements ॥

\section{3-5. Filtre || enhancement edge \| ou filtre à || réhaussement ॥}

L'Ilenhancement edge filter" ou filtre à "réhaussement " de contours est un filtre graphique que l'on applique pour améliorer les contours d'une image, en l'occurrence la carte des zones anomales de II dérangements II. Ce filtre permet d'améliorer la qualité d'une image en accentuant la visibilité de ses détails. Le "r réhaussement II des images permet de faciliter l'interprétation visuelle. Les images numériques ont l'avantage de nous permettre de manipuler assez facilement les valeurs enregistrées pour chaque pixel. Un filtre à " réhaussement ॥ de contours souligne et accentue les zones de discontinuités brusques et abruptes tels les changements de faciès dus à des changements brutaux de densités (Figure 9 ) [13]. II a été utilisé un If filtre à réhaussement II de type laplacien dont la sortie s'exprime par l'expression suivante :

$$
I(x, y)-k L(x, y) \text { ou } I_{i, j}-k L_{i, j} \text { et } 0<k \leq 1
$$

La forme discrète de l'expression du laplacien-filtre peut être mise sous la forme suivante [14] :

$$
\left.L_{i, j}=\mid I_{i+1, j}-2 I_{i, j}+I_{i-1, j}\right]+\left[I_{i, j+1}-2 I_{i, j}+I_{i, j-1}\right]=-5 \mid I_{i, j}-\frac{1}{5}\left[I_{i+1, j}+I_{i-1, j}+I_{i, j}+I_{i, j+1}+I_{i, j-1} \mid\right)
$$


DIRECTION NORD (DISTANCE EN METRES)

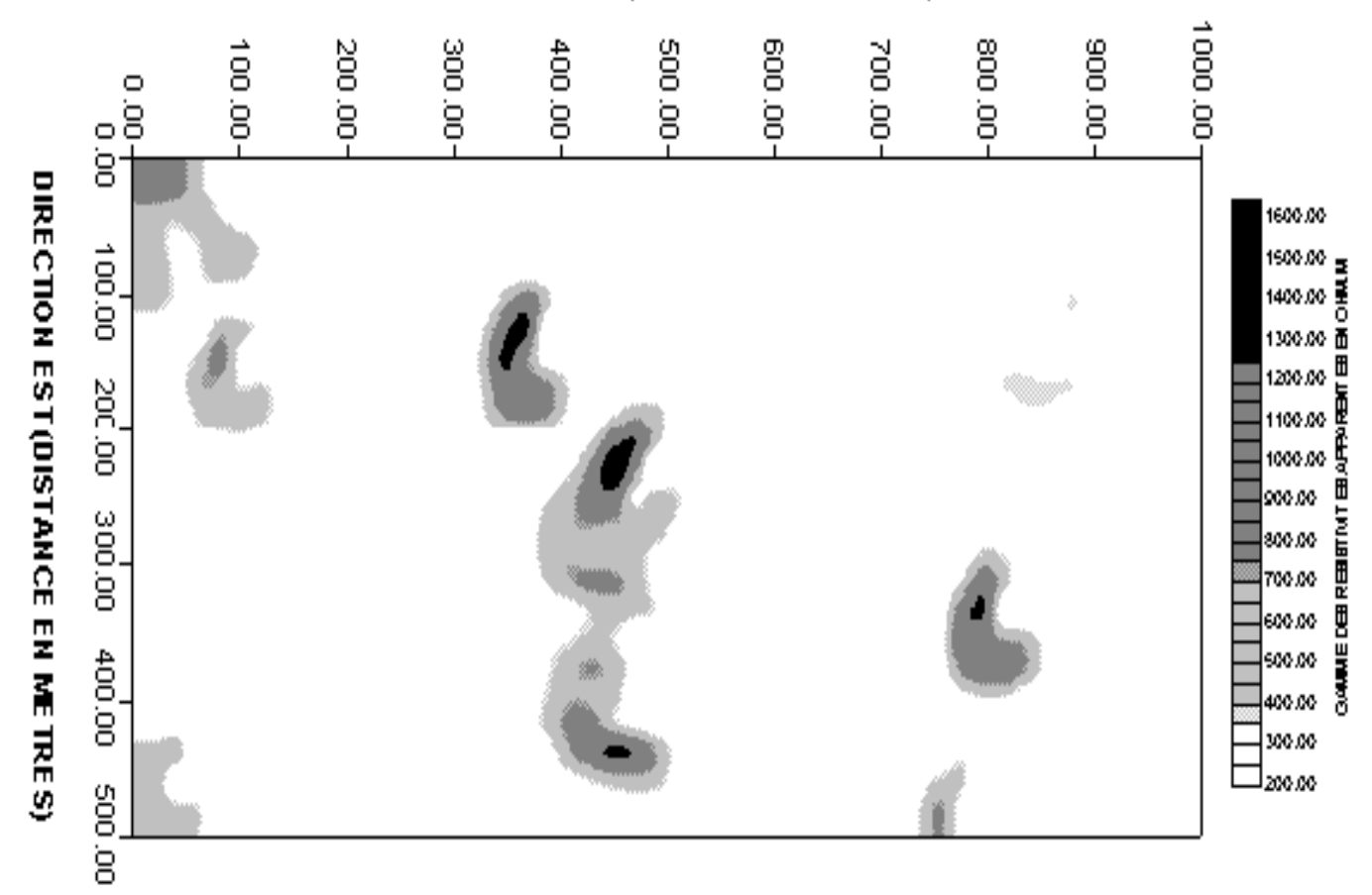

Figure 9 : Réponse du filtre à II réhaussement II laplacien appliqué aux zones anomales de "I dérangements "

\section{Résultats et discussion}

Le filtrage spatial de la carte des résistivités anomales a été réalisé avec le logiciel EasyMapping [15]. La carte des résistivités anomales (Figure 6 ) donne pour la zone d'étude les valeurs de la résistivité apparente obtenues pour le dispositif Schlumberger centré. La carte de résistivité fournit une interprétation directe et imagée des séries de profils de résistivités dont elle découle. La carte de résistivité apparente permet d'identifier les zones anomales coïncidant fortement avec les I dérangements ॥.

Les zones anomales de "dérangements " détectées en surface présentent une distribution aléatoire (Figure 7). Le filtre à " réhaussement " de type laplacien a été utilisé pour filtrer la carte des résistivités anomales. Les zones anomales des "dérangements " (Figure 9 ) ont été nettement circonscrites par des contours très nets : une bonne corrélation se manifeste par rapport à la distribution des zones de "I dérangements II mises en évidence par la cartographie géoélectrique (Figure 6 ). Le filtre à "réhaussement " présente la particularité de filtrer le signal géophysique 
origine : les contours des zones anomales de II dérangements " délimitent des zones de " dérangements " à intensité variable. Cette singularité se traduit par une variation croissante de la résistivité électrique au sein même de la zone de "I dérangements I. Cette caractéristique pourrait servir efficacement d'indicateur du niveau de "I dérangement ॥ au sein même des séries phosphatées dites I dérangées "I.

La carte de la réponse du laplacien-filtre passe-haut (Figure 8 ) décrit la distribution aléatoire des zones de II dérangements $\|$ : elle atteste du zoning des fortes variations latérales de densité elles-même indicatrices de ces zones de "dérangements I. Les zones de II dérangements II supposés formés de blocs homogènes, tels que corrélés par la réponse du filtre à " réhaussement " (Figure 9 ) se présentent sous forme d'amas aléatoirement dispersés de tailles moindres: la carte du laplacien-filtre passe-haut confirme cette singularité de la nature des $\|$ dérangements $\|$ et manifeste globalement une certaine et efficace correspondance avec la carte de la distribution des zones anomales de celle du filtre à II réhaussement II. La carte de la réponse du laplacien-filtre pourrait à juste titre être considérée comme une carte de détails destinée aux calculs prédictifs des surfaces des zones de II dérangements $\|$.

\section{Conclusion}

Cette étude pilote singulière a permis de donner une réponse analytique à l'analyse d'une carte de données géophysiques dans le domaine minier. La méthode géoélectrique utilisée dans cette approche originale a fourni des résultats satisfaisants tout en confirmant l'adaptation de la méthode des résistivités.

Les zones anomales correspondant aux "dérangements" sont assimilées aux distributions anormales de densité. Les utilitaires classiques de traitement de cartes dans le domaine spatial se sont avérés efficaces sous les hypothèses énoncées. Les différentes cartes transformées confirment les limites des contours des zones anomales détectées par la méthode géoélectrique. Les cartes du filtre à " réhaussement "I et du laplacien-filtre passe-haut soulignent les variations du contratse de densité entre les zones anomales de II dérangements $\|$ et les zones phosphatées saines.

L'analyse spatiale a permis de traiter les anomalies de la résistivité comme des discontinuités. Elle a d'ailleurs permis de confirmer la distribution aléatoire des "dérangements" sur la zone d'étude. Les cartes transformées permettent également aux géomètres-topographes de circonscrire efficacement les surfaces anomales des réserves des phosphates. 


\section{Références}

[1] - A. Kchikach, M. Jaffal, T. Aifa et L. Bahi, "I Cartographie de corps stériles sous couverture quaternaire par méthode de résistivités électriques dans le gisement phosphaté de Sidi Chennane (Maroc) II, CR, Géosciences, 334 (2002) 379-386

[2] - S. Bakkali et L. Bahi, "Cartographie des II dérangements ॥ de séries phosphatées par mesures de résistivités électriques II, Journal des Sciences Pour l'Ingénieur, J.S.P.I., édité par l'Ecole Supérieure Polytechnique - Dakar, ISSN 0851-4453, Vol. 6 (2006) $1-10$

[3] - A. Michard, II Les phosphates II, Notes \& Mémoires, №276 (1975), édité par le Service Géologique du Maroc

[4] - S .Bakkali et J. Bouyalaoui, "Essai d'optimisation de la capacité de retenue d'eau d'un lac par caractérisation géophysique du recouvrement argileux II, African Journal of Science \& Technology, AJST, 5 (2) (2005) 12-22

[5] - S. Bakkali et J. Bouyalaoui, "I Prospection géophysique appliquée à l'évaluation des eaux souterraines de Mediouna (Tanger, Maroc) II, Journal des Sciences Pour I'Ingénieur, J.S.P.I., 4 (2004) 13-22

[6] - S. Bakkali et J. Bouyalaoui, "Contribution de la prospection électrique à la connaissance des ressources en eau du village d'Anergui (Tafraoute, Anti-Atlas Marocain II, e-Gnosis, [online], 3,5 (2005), www.e-gnosis.udg.mx/vol3/art5

[7] - A. Dey and H. F. Morrinson, II Resistivity modelling for arbitrarily shaped twodimensional structures II, Geophysical Prospecting, 27 (1979) 106-136

[8] - S. Bakkali II A resistivity survey of phosphate deposits containing hardpan pockets in Oulad Abdoun, Moroccoll, Geofisica Internacional, 45 (1) (2006) 73-82

[9] - S. Rakotoniaina, "Iraitement des anomalies en géophysiquell, Thèse d'Université, Institut et Observatoire d' Antananarivo (I.O.G.A), Université d'Antananarivo, Madagascar (1999)

[10] - P. Charbonnier, II Reconstruction d'image : régularisation avec prise en compte des discontinuités II, Thèse de Doctorat, Université de Nice Sophia-Antipolis, (1994)

[11]- P. Fedi and G. Florio, "Detection of potential fields boundaries by enhanced horizontal derivative methodII, Geophysical Prospecting, 49 (2001) 40-58

[12] - G. Cooper and D. Cowan, "The application of fractional calculus to potential field data II, Exploration Geophysics, 34 (2003) 51-56

[13] - Z. Shi and G. Butt, "New enhancement filters for geological mapping ", ASEG $17^{\text {th }}$ Geophysical Conference and Exhibition (2004) Sydney, Extended Abstracts.

[14] - C. Garnica, F. Boochs and M. Twardochlib "I new approach to edge-preserving smoothing for edge extraction and image segmentation II, International Archives of Photogrammetry and Remote Sensing (IAPRS), Amsterdam, Vol XXXIII (2000)

[15] - O. Monnereau, "About EasyMapping software version 3.0, Manuel d'utilisation", Copyright 2001-2003, Logiciel EasyMapping, Olivier Monnereau (2003). 